\title{
Article \\ The Existence and Uniquenes Solution of Nonlinear Integral Equations via Common Fixed Point Theorems
}

\author{
Gunaseelan Mani ${ }^{1}\left(\mathbb{D}\right.$, Arul Joseph Gnanaprakasam ${ }^{2}{ }^{\oplus}$, Yongjin $\mathrm{Li}^{3}$ and Zhaohui $\mathrm{Gu}^{4, *}$ \\ 1 Department of Mathematics, Sri Sankara Arts and Science College (Autonomous), Madras University, \\ Enathur, Kanchipuram 631 561, Tamil Nadu, India; mathsguna@yahoo.com \\ 2 Department of Mathematics, College of Engineering and Technology, Faculty of Engineering and Technology, \\ SRM Institute of Science and Technology, SRM Nagar, Kattankulathur 603 203, Tamil Nadu, India; \\ aruljoseph.alex@gmail.com \\ 3 Department of Mathematics, Sun Yat-Sen University, Guangzhou 510275, China; stslyj@mail.sysu.edu.cn \\ 4 School of Mathematics and Statistics, Guangdong University of Foreign Studies, Guangzhou 510006, China \\ * Correspondence: zhgugz@163.com
}

check for updates

Citation: Mani, G.; Gnanaprakasam, A.J.; Li, Y.; Gu, Z. The Existence and Uniquenes Solution of Nonlinear Integral Equations via Common Fixed Point Theorems. Mathematics 2021, 9, 1179. https://doi.org/ $10.3390 /$ math9111179

Academic Editor: Pasquale Vetro

Received: 20 April 2021

Accepted: 18 May 2021

Published: 24 May 2021

Publisher's Note: MDPI stays neutral with regard to jurisdictional claims in published maps and institutional affiliations.

Copyright: (c) 2021 by the authors. Licensee MDPI, Basel, Switzerland. This article is an open access article distributed under the terms and conditions of the Creative Commons Attribution (CC BY) license (https:// creativecommons.org/licenses/by/ $4.0 /)$

\begin{abstract}
In this paper, we prove some common fixed-point theorems on complex partial metric space. The presented results generalize and expand some of the well-known results in the literature. We also explore some of the applications of our key results.
\end{abstract}

Keywords: integral equations; complex partial metric space; common fixed point

MSC: 47H10; 54H25

\section{Introduction}

Azam et al. [1] introduced the concept of complex-valued metric spaces and studied some fixed point theorems for mappings satisfying a rational inequality.

Two years later, in [2], Rao et al. discussed for the first time the idea of complex-valued b-metric spaces.

In 2017, Dhivya and Marudai [3] introduced the concept of complex partial metric space and suggested a plan to expand the results, as well as proving common fixed-point theorems under the rational expression contraction condition. This idea has been followed by Gunaseelan [4], who introduced the concept of complex partial b-metric spaces and discussed some results of fixed-point theory for self-mappings in these new spaces.

In [5], Prakasam and Gunaseelan proved the existence and uniqueness of a common fixed-point (with an illustrative example) theorem using CLR and E.A. properties in complex partial b-metric spaces. Their proved results generalize and extend some of the well-known results in the literature.

In [6], Gunaseelan et al. proved a fixed-point theorem in complex partial b-metric spaces under a contraction mapping. They also gave some applications of their main results.

In this paper, we prove some common fixed-point theorems on complex partial metric space.

\section{Preliminaries}

Let $\mathfrak{C}$ be the set of complex numbers and $\tau_{1}, \tau_{2}, \tau_{3} \in \mathfrak{C}$. Define a partial order $\preceq$ on $\mathfrak{C}$ as follows:

$\tau_{1} \preceq \tau_{2}$ if and only if $\mathcal{R}\left(\tau_{1}\right) \leq \mathcal{R}\left(\tau_{2}\right), \mathcal{I}\left(\tau_{1}\right) \leq \mathcal{I}\left(\tau_{2}\right)$.

Consequently, one can infer that $\tau_{1} \preceq \tau_{2}$ if one of the following conditions is satisfied:

(i) $\mathcal{R}\left(\tau_{1}\right)=\mathcal{R}\left(\tau_{2}\right), \mathcal{I}\left(\tau_{1}\right)<\mathcal{I}\left(\tau_{2}\right)$,

(ii) $\mathcal{R}\left(\tau_{1}\right)<\mathcal{R}\left(\tau_{2}\right), \mathcal{I}\left(\tau_{1}\right)=\mathcal{I}\left(\tau_{2}\right)$,

(iii) $\mathcal{R}\left(\tau_{1}\right)<\mathcal{R}\left(\tau_{2}\right), \mathcal{I}\left(\tau_{1}\right)<\mathcal{I}\left(\tau_{2}\right)$, 
(iv) $\mathcal{R}\left(\tau_{1}\right)=\mathcal{R}\left(\tau_{2}\right), \mathcal{I}\left(\tau_{1}\right)=\mathcal{I}\left(\tau_{2}\right)$.

In particular, we write $\tau_{1} \precsim \tau_{2}$ if $\tau_{1} \neq \tau_{2}$, and one of $(i)$, (ii) and (iii) is satisfied and we write $\tau_{1} \prec \tau_{2}$ if only (iii) is satisfied. Notice that

(a) If $0 \preceq \tau_{1} \precsim \tau_{2}$, then $\left|\tau_{1}\right|<\left|\tau_{2}\right|$,

(b) If $\tau_{1} \preceq \tau_{2}$ and $\tau_{2} \prec \tau_{3}$, then $\tau_{1} \prec \tau_{3}$,

(c) If $\eta, \gamma \in \mathbb{R}$ and $\eta \leq \gamma$, then $\eta \tau_{1} \preceq \gamma \tau_{1}$ for all $0 \preceq \tau_{1} \in \mathfrak{C}$.

Here $\mathfrak{C}_{+}\left(=\left\{(\aleph, \mathfrak{y}) \mid \aleph, \mathfrak{y} \in \mathbb{R}_{+}\right\}\right)$and $\mathbb{R}_{+}(=\{\aleph \in \mathbb{R} \mid \aleph \geq 0\})$ denote the set of nonnegative complex numbers and the set of non negative real numbers, respectively.

Now, let us recall some basic concepts and notations that will be used below.

Definition 1 ([3]). A complex partial metric on a non-void set $G$ is a function $\varrho_{c b}: G \times G \rightarrow \mathbb{C}^{+}$ such that for all $\theta, \omega, \vartheta \in G$ :

(i) $\quad 0 \preceq \varrho_{c b}(\theta, \theta) \preceq \varrho_{c b}(\theta, \omega)$ (small self-distances)

(ii) $\varrho_{c b}(\theta, \omega)=\varrho_{c b}(\omega, \theta)$ (symmetry)

(iii) $\varrho_{c b}(\theta, \theta)=\varrho_{c b}(\theta, \omega)=\varrho_{c b}(\omega, \omega)$ if and only if $\theta=\omega$ (equality)

(iv) $\varrho_{c b}(\theta, \omega) \preceq \varrho_{c b}(\theta, \vartheta)+\varrho_{c b}(\vartheta, \omega)-\varrho_{c b}(\vartheta, \vartheta)$ (triangularity).

A complex partial metric space is a pair $\left(G, \varrho_{c b}\right)$ such that $G$ is a non-void set and $\varrho_{c b}$ is the complex partial metric on $G$.

Definition 2 ([3]). Let $\left(G, \wp_{c b}\right)$ be a complex partial metric space. Let $\left\{\theta_{n}\right\}$ be any sequence in G. Then

(i) The sequence $\left\{\theta_{n}\right\}$ is said to converge to $\theta$, if $\lim _{n \rightarrow \infty} \wp_{c b}\left(\theta_{n}, \theta\right)=\wp_{c b}(\theta, \theta)$.

(ii) The sequence $\left\{\theta_{n}\right\}$ is said to be a Cauchy sequence in $\left(G, \wp_{c b}\right)$ if $\lim _{n, m \rightarrow \infty} \wp_{c b}\left(\theta_{n}, \theta_{m}\right)$ exists and is finite.

(iii) $\left(G, \wp_{c b}\right)$ is said to be a complete complex partial metric space if for every Cauchy sequence $\left\{\theta_{n}\right\}$ in $G$ there exists $\theta \in G$ such that $\lim _{n, m \rightarrow \infty} \wp_{c b}\left(\theta_{n}, \theta_{m}\right)=\lim _{n \rightarrow \infty} \wp_{c b}\left(\theta_{n}, \theta\right)=\wp_{c b}(\theta, \theta)$.

(iv) A mapping $\Pi: G \rightarrow G$ is said to be continuous at $\theta_{0} \in G$ if for every $\epsilon>0$, there exists $\delta>0$ such that $\Pi\left(B_{\wp_{c b}}\left(\theta_{0}, \delta\right)\right) \subset B_{\wp_{c b}}\left(\Pi\left(\theta_{0}, \epsilon\right)\right)$.

Definition 3 ([3]). Let $\Pi$ and $\Psi$ be self-mappings of non-void set $G$. A point $\aleph \in G$ is called a common fixed point of $\Pi$ and $\Psi$ if $\aleph=\Pi \aleph=\Psi \aleph$.

Theorem 1 ([3]). Let $(G, \preceq)$ be a partially ordered set and suppose that there exists a complex partial metric $\varrho_{c b}$ in $G$ such that $\left(G, \varrho_{c b}\right)$ is a complete complex partial metric space. Let $\Pi, \Psi$ : $G \rightarrow G$ be a pair of weakly increasing mappings, and suppose that for every comparable $\aleph, \mathfrak{y} \in G$ we have either

$$
\varrho_{c b}(\Pi \aleph, \Psi \mathfrak{y}) \preceq a \frac{\varrho_{c b}(\aleph, \Pi \aleph) \varrho_{c b}(\mathfrak{y}, \Psi \mathfrak{y})}{\varrho_{c b}(\aleph, \mathfrak{y})}+b \varrho_{c b}(\aleph, \mathfrak{y})
$$

for $\varrho_{c b}(\aleph, \mathfrak{y}) \neq 0$ with $a \geq 0, b \geq 0, a+b<1$, or

$$
\varrho_{c b}(\Pi \aleph, \Psi \mathfrak{y})=0 \text { if } \varrho_{c b}(\aleph, \mathfrak{y})=0 .
$$

If $\Pi$ or $\Psi$ is continuous, then $\Pi$ and $\Psi$ have a common fixed point $\alpha \in G$ and $\varrho_{c b}(\alpha, \alpha)=0$.

Inspired by Theorem 1, here we prove some common fixed-point theorems on complex partial metric space with an application. For complex partial metric space, we will use the CPMS notation.

\section{Main Results}

Theorem 2. Let $\left(G, \wp_{c b}\right)$ be a complete CPMS and $\Pi, \Psi: G \rightarrow G$ be two continuous mappings such that 


$$
\begin{aligned}
& \wp_{c b}(\Pi \theta, \Psi \omega) \preceq \curlywedge \max \left\{\wp_{c b}(\theta, \omega), \wp_{c b}(\theta, \Pi \theta), \wp_{c b}(\omega, \Psi \omega),\right. \\
&\left.\frac{1}{2}\left(\wp_{c b}(\theta, \Psi \omega)+\wp_{c b}(\omega, \Pi \theta)\right)\right\},
\end{aligned}
$$

for all $\theta, \omega \in G$, where $0 \leq \curlywedge<1$ and $\wp_{c b}(\Pi \theta, \Psi \omega) \neq 0$. Then, the pair $(\Pi, \Psi)$ has a unique common fixed point and $\wp_{c b}\left(\theta^{*}, \theta^{*}\right)=0$.

Proof. Let $\theta_{0}$ be arbitrary point in $G$ and define a sequence $\left\{\theta_{n}\right\}$ as follows:

$$
\theta_{2 n+1}=\Pi \theta_{2 n} \text { and } \theta_{2 n+2}=\Psi \theta_{2 n+1}, n=0,1,2, \ldots
$$

Then by (1) and (2), we obtain

$$
\begin{aligned}
\wp_{c b}\left(\theta_{2 n+1}, \theta_{2 n+2}\right)= & \wp_{c b}\left(\Pi \theta_{2 n}, \Psi \theta_{2 n+1}\right) \\
\preceq & \curlywedge \max \left\{\wp_{c b}\left(\theta_{2 n}, \theta_{2 n+1}\right), \wp_{c b}\left(\theta_{2 n}, \Pi \theta_{2 n}\right), \wp_{c b}\left(\theta_{2 n+1}, \Psi \theta_{2 n+1}\right),\right. \\
& \left.\frac{1}{2}\left(\wp_{c b}\left(\theta_{2 n}, \Psi \theta_{2 n+1}\right)+\wp_{c b}\left(\theta_{2 n+1}, \Pi \theta_{2 n}\right)\right)\right\} \\
\preceq & \curlywedge \max \left\{\wp_{c b}\left(\theta_{2 n}, \theta_{2 n+1}\right), \wp_{c b}\left(\theta_{2 n}, \theta_{2 n+1}\right), \wp_{c b}\left(\theta_{2 n+1}, \theta_{2 n+2}\right),\right. \\
& \left.\frac{1}{2}\left(\wp_{c b}\left(\theta_{2 n}, \theta_{2 n+2}\right)+\wp_{c b}\left(\theta_{2 n+1}, \theta_{2 n+1}\right)\right)\right\} \\
\preceq & \curlywedge \max \left\{\wp_{c b}\left(\theta_{2 n}, \theta_{2 n+1}\right), \wp_{c b}\left(\theta_{2 n+1}, \theta_{2 n+2}\right),\right. \\
& \frac{1}{2}\left(\wp_{c b}\left(\theta_{2 n}, \theta_{2 n+1}\right)+\wp_{c b}\left(\theta_{2 n+1}, \theta_{2 n+2}\right)-\wp_{c b}\left(\theta_{2 n+1}, \theta_{2 n+1}\right)\right. \\
& \left.\left.+\wp_{c b}\left(\theta_{2 n+1}, \theta_{2 n+1}\right)\right)\right\} \\
= & \curlywedge \max \left\{\wp_{c b}\left(\theta_{2 n}, \theta_{2 n+1}\right), \wp_{c b}\left(\theta_{2 n+1}, \theta_{2 n+2}\right),\right. \\
& \left.\frac{1}{2}\left(\wp_{c b}\left(\theta_{2 n}, \theta_{2 n+1}\right)+\wp_{c b}\left(\theta_{2 n+1}, \theta_{2 n+2}\right)\right)\right\}
\end{aligned}
$$

\section{Case I:}

If $\max \left\{\wp_{c b}\left(\theta_{2 n}, \theta_{2 n+1}\right), \wp_{c b}\left(\theta_{2 n+1}, \theta_{2 n+2}\right), \frac{1}{2}\left(\wp_{c b}\left(\theta_{2 n}, \theta_{2 n+1}\right)+\wp_{c b}\left(\theta_{2 n+1}, \theta_{2 n+2}\right)\right)\right\}=\wp_{c b}$ $\left(\theta_{2 n+1}, \theta_{2 n+2}\right)$, then we have

$$
\wp_{c b}\left(\theta_{2 n+1}, \theta_{2 n+2}\right) \preceq \curlywedge \wp_{c b}\left(\theta_{2 n+1}, \theta_{2 n+2}\right) .
$$

This implies $\curlywedge \geq 1$, which is a contradiction.

\section{Case II:}

If $\max \left\{\wp_{c b}\left(\theta_{2 n}, \theta_{2 n+1}\right), \wp_{c b}\left(\theta_{2 n+1}, \theta_{2 n+2}\right), \frac{1}{2}\left(\wp_{c b}\left(\theta_{2 n}, \theta_{2 n+1}\right)+\wp_{c b}\left(\theta_{2 n+1}, \theta_{2 n+2}\right)\right)\right\}=\wp_{c b}$ $\left(\theta_{2 n}, \theta_{2 n+1}\right)$, then we have

$$
\wp_{c b}\left(\theta_{2 n+1}, \theta_{2 n+2}\right) \preceq \curlywedge \wp_{c b}\left(\theta_{2 n}, \theta_{2 n+1}\right) .
$$

From the next step, we have

$$
\begin{aligned}
& \wp_{c b}\left(\theta_{2 n+2}, \theta_{2 n+3}\right) \preceq \curlywedge \max \left\{\wp_{c b}\left(\theta_{2 n+1}, \theta_{2 n+2}\right), \wp_{c b}\left(\theta_{2 n+2}, \theta_{2 n+3}\right),\right. \\
&\left.\frac{1}{2}\left(\wp_{c b}\left(\theta_{2 n+1}, \theta_{2 n+2}\right)+\wp_{c b}\left(\theta_{2 n+2}, \theta_{2 n+3}\right)\right)\right\} .
\end{aligned}
$$

The following three cases arise.

\section{Case IIa:}

$$
\wp_{c b}\left(\theta_{2 n+2}, \theta_{2 n+3}\right) \preceq \curlywedge \wp_{c b}\left(\theta_{2 n+2}, \theta_{2 n+3}\right),
$$

which implies $\curlywedge \geq 1$, which is a contradiction. 


\section{Case IIb:}

$$
\wp_{c b}\left(\theta_{2 n+2}, \theta_{2 n+3}\right) \preceq \curlywedge \frac{1}{2}\left(\wp_{c b}\left(\theta_{2 n+1}, \theta_{2 n+2}\right)+\wp_{c b}\left(\theta_{2 n+2}, \theta_{2 n+3}\right)\right) .
$$

This implies that

$$
\wp_{c b}\left(\theta_{2 n+2}, \theta_{2 n+3}\right) \preceq \frac{\curlywedge}{(2-\curlywedge)} \wp_{c b}\left(\theta_{2 n+1}, \theta_{2 n+2}\right) .
$$

Since $a:=\frac{\curlywedge}{2-\curlywedge}<1$, we get $\wp_{c b}\left(\theta_{n+1}, \theta_{n+2}\right) \preceq a \wp_{c b}\left(\theta_{n}, \theta_{n+1}\right)$. Therefore $\left\{\theta_{n}\right\}_{n \in \mathbb{N}}$ is a Cauchy sequence in $G$.

\section{Case IIc:}

$$
\wp_{c b}\left(\theta_{2 n+2}, \theta_{2 n+3}\right) \preceq \curlywedge \wp_{c b}\left(\theta_{2 n+1}, \theta_{2 n+2}\right) .
$$

From (3) and (5), $\forall n=0,1,2, \ldots$, we get

$$
\wp_{c b}\left(\theta_{n+1}, \theta_{n+2}\right) \preceq \curlywedge \wp_{c b}\left(\theta_{n}, \theta_{n+1}\right) \preceq \ldots \preceq \curlywedge^{n+1} \wp_{c b}\left(\theta_{0}, \theta_{1}\right) .
$$

For $m, n \in \mathbb{N}$, with $m>n$, we have

$$
\begin{aligned}
& \wp_{c b}\left(\theta_{n}, \theta_{m}\right) \preceq \wp_{c b}\left(\theta_{n}, \theta_{n+1}\right)+\wp_{c b}\left(\theta_{n+1}, \theta_{m}\right)-\wp_{c b}\left(\theta_{n+1}, \theta_{n+1}\right) \\
& \preceq \wp_{c b}\left(\theta_{n}, \theta_{n+1}\right)+\wp_{c b}\left(\theta_{n+1}, \theta_{m}\right) \\
& \preceq \wp_{c b}\left(\theta_{n}, \theta_{n+1}\right)+\wp_{c b}\left(\theta_{n+1}, \theta_{n+2}\right)+\wp_{c b}\left(\theta_{n+2}, \theta_{m}\right) \\
&-\wp_{c b}\left(\theta_{n+2}, \theta_{n+2}\right) \\
& \preceq \wp_{c b}\left(\theta_{n}, \theta_{n+1}\right)+\wp_{c b}\left(\theta_{n+1}, \theta_{n+2}\right)+\wp_{c b}\left(\theta_{n+2}, \theta_{m}\right) \\
& \preceq \wp_{c b}\left(\theta_{n}, \theta_{n+1}\right)+\wp_{c b}\left(\theta_{n+1}, \theta_{n+2}\right)+\wp_{c b}\left(\theta_{n+2}, \theta_{n+3}\right) \\
&+\ldots+\wp_{c b}\left(\theta_{m-2}, \theta_{m-1}\right)+\wp_{c b}\left(\theta_{m-1}, \theta_{m}\right) .
\end{aligned}
$$

Moreover, by using (5), we get

$$
\begin{aligned}
\wp_{c b}\left(\theta_{n}, \theta_{m}\right) \preceq & \curlywedge^{n} \wp_{c b}\left(\theta_{0}, \theta_{1}\right)+\curlywedge^{n+1} \wp_{c b}\left(\theta_{0}, \theta_{1}\right)+\curlywedge^{n+2} \wp_{c b}\left(\theta_{0}, \theta_{1}\right) \\
& +\ldots+\curlywedge^{m-2} \wp_{c b}\left(\theta_{0}, \theta_{1}\right)+\curlywedge^{m-1} \wp_{c b}\left(\theta_{0}, \theta_{1}\right) \\
= & \sum_{i=1}^{m-n} \curlywedge^{i+n-1} \wp_{c b}\left(\theta_{0}, \theta_{1}\right) .
\end{aligned}
$$

Therefore

$$
\begin{aligned}
\left|\wp_{c b}\left(\theta_{n}, \theta_{m}\right)\right| & \leq \sum_{i=1}^{m-n} \curlywedge^{i+n-1}\left|\wp_{c b}\left(\theta_{0}, \theta_{1}\right)\right|=\sum_{t=n}^{m-1} \curlywedge^{t}\left|\wp_{c b}\left(\theta_{0}, \theta_{1}\right)\right| \\
& \leq \sum_{i=n}^{\infty}\left|\wp_{c b}\left(\theta_{0}, \theta_{1}\right)\right| \\
& =\frac{\curlywedge^{n}}{1-\curlywedge}\left|\wp_{c b}\left(\theta_{0}, \theta_{1}\right)\right| .
\end{aligned}
$$

Then, we have

$$
\left|\wp_{c b}\left(\theta_{n}, \theta_{m}\right)\right| \leq \frac{\curlywedge^{n}}{1-\curlywedge}\left|\wp_{c b}\left(\theta_{0}, \theta_{1}\right)\right| \rightarrow 0 \quad \text { as } \quad n \rightarrow \infty .
$$

Hence, $\left\{\theta_{n}\right\}$ is a Cauchy sequence in $G$. 


\section{Case III:}

If $\max \left\{\wp_{c b}\left(\theta_{2 n}, \theta_{2 n+1}\right), \wp_{c b}\left(\theta_{2 n+1}, \theta_{2 n+2}\right), \frac{1}{2}\left(\wp_{c b}\left(\theta_{2 n}, \theta_{2 n+1}\right)+\wp_{c b}\left(\theta_{2 n+1}, \theta_{2 n+2}\right)\right)\right\}=$ $\frac{1}{2}\left(\wp_{c b}\left(\theta_{2 n}, \theta_{2 n+1}\right)+\wp_{c b}\left(\theta_{2 n+1}, \theta_{2 n+2}\right)\right)$.

Then, we have

$$
\wp_{c b}\left(\theta_{2 n+1}, \theta_{2 n+2}\right) \preceq \frac{\curlywedge}{2}\left(\wp_{c b}\left(\theta_{2 n}, \theta_{2 n+1}\right)+\wp_{c b}\left(\theta_{2 n+1}, \theta_{2 n+2}\right)\right)
$$

Hence,

$$
\wp_{c b}\left(\theta_{2 n+1}, \theta_{2 n+2}\right) \preceq \frac{\curlywedge}{2-\curlywedge} \wp_{c b}\left(\theta_{2 n}, \theta_{2 n+1}\right) .
$$

For the next step, we have

$$
\begin{aligned}
\wp_{c b}\left(\theta_{2 n+2}, \theta_{2 n+3}\right) \preceq & \curlywedge \max \left\{\wp_{c b}\left(\theta_{2 n+1}, \theta_{2 n+2}\right), \wp_{c b}\left(\theta_{2 n+2}, \theta_{2 n+3}\right),\right. \\
& \left.\frac{1}{2}\left(\wp_{c b}\left(\theta_{2 n+1}, \theta_{2 n+2}\right)+\wp_{c b}\left(\theta_{2 n+2}, \theta_{2 n+3}\right)\right)\right\} .
\end{aligned}
$$

Then, we have the following three cases:

\section{Case IIIa:}

$$
\wp_{c b}\left(\theta_{2 n+2}, \theta_{2 n+3}\right) \preceq \curlywedge \wp_{c b}\left(\theta_{2 n+2}, \theta_{2 n+3}\right),
$$

which implies $\curlywedge \geq 1$, which is a contradiction.

Case IIIb:

$$
\wp_{c b}\left(\theta_{2 n+2}, \theta_{2 n+3}\right) \preceq \curlywedge \wp_{c b}\left(\theta_{2 n+1}, \theta_{2 n+2}\right) .
$$

Then by (6) and (7), we get $\wp_{c b}\left(\theta_{n+1}, \theta_{n+2}\right) \preceq \gamma_{\wp_{c b}}\left(\theta_{n}, \theta_{n+1}\right)$, where $\gamma=\max \left\{\curlywedge, \frac{\curlywedge}{2-\curlywedge}\right\}<1$. Hence $\left\{\theta_{n}\right\}_{n \in \mathbb{N}}$ is a Cauchy sequence in $G$.

Case IIIc:

$$
\wp_{c b}\left(\theta_{2 n+2}, \theta_{2 n+3}\right) \preceq \frac{1}{2}\left(\wp_{c b}\left(\theta_{2 n+1}, \theta_{2 n+2}\right)+\wp_{c b}\left(\theta_{2 n+2}, \theta_{2 n+3}\right)\right) .
$$

Hence, we obtain

$$
\wp_{c b}\left(\theta_{2 n+2}, \theta_{2 n+3}\right) \preceq \frac{\curlywedge}{(2-\curlywedge)} \wp_{c b}\left(\theta_{2 n+1}, \theta_{2 n+2}\right) .
$$

Using (6) and (8) yields

$$
\wp_{c b}\left(\theta_{n+1}, \theta_{n+2}\right) \preceq 2 \wp_{c b}\left(\theta_{n}, \theta_{n+1}\right),
$$

where $0 \leq \imath=\frac{\curlywedge}{2-\ell}<1$.

Then, $\forall n=0,1,2, \ldots$, and we get

$$
\wp_{c b}\left(\theta_{n+1}, \theta_{n+2}\right) \preceq 2 \wp_{c b}\left(\theta_{n}, \theta_{n+1}\right) \preceq \ldots \preceq 2^{n+1} \wp_{c b}\left(\theta_{0}, \theta_{1}\right) .
$$


For $m, n \in \mathbb{N}$, with $m>n$, we have

$$
\begin{aligned}
& \wp_{c b}\left(\theta_{n}, \theta_{m}\right) \preceq \wp_{c b}\left(\theta_{n}, \theta_{n+1}\right)+\wp_{c b}\left(\theta_{n+1}, \theta_{m}\right)-\wp_{c b}\left(\theta_{n+1}, \theta_{n+1}\right) \\
& \preceq \wp_{c b}\left(\theta_{n}, \theta_{n+1}\right)+\wp_{c b}\left(\theta_{n+1}, \theta_{m}\right) \\
& \preceq \wp_{c b}\left(\theta_{n}, \theta_{n+1}\right)+\wp_{c b}\left(\theta_{n+1}, \theta_{n+2}\right)+\wp_{c b}\left(\theta_{n+2}, \theta_{m}\right) \\
& -\wp_{c b}\left(\theta_{n+2}, \theta_{n+2}\right) \\
& \preceq \wp_{c b}\left(\theta_{n}, \theta_{n+1}\right)+\wp_{c b}\left(\theta_{n+1}, \theta_{n+2}\right)+\wp_{c b}\left(\theta_{n+2}, \theta_{m}\right) \\
& \preceq \wp_{c b}\left(\theta_{n}, \theta_{n+1}\right)+\wp_{c b}\left(\theta_{n+1}, \theta_{n+2}\right)+\wp_{c b}\left(\theta_{n+2}, \theta_{n+3}\right) \\
& \left.+\ldots+\wp_{c b}\left(\theta_{m-2}, \theta_{m-1}\right)\right)+\wp_{c b}\left(\theta_{m-1}, \theta_{m}\right) \text {. }
\end{aligned}
$$

Using (9), we get

$$
\begin{aligned}
\wp_{c b}\left(\theta_{n}, \theta_{m}\right) \preceq & 2^{n} \wp_{c b}\left(\theta_{0}, \theta_{1}\right)+2^{n+1} \wp_{c b}\left(\theta_{0}, \theta_{1}\right)+2^{n+2} \wp_{c b}\left(\theta_{0}, \theta_{1}\right) \\
& +\ldots+2^{m-2} \wp_{c b}\left(\theta_{0}, \theta_{1}\right)+2^{m-1} \wp_{c b}\left(\theta_{0}, \theta_{1}\right) \\
= & \sum_{i=1}^{m-n} 2^{i+n-1} \wp_{c b}\left(\theta_{0}, \theta_{1}\right) .
\end{aligned}
$$

Therefore,

$$
\begin{aligned}
\left|\wp_{c b}\left(\theta_{n}, \theta_{m}\right)\right| & \leq \sum_{i=1}^{m-n} 2^{i+n-1}\left|\wp_{c b}\left(\theta_{0}, \theta_{1}\right)\right|=\sum_{t=n}^{m-1} 2^{t}\left|\wp_{c b}\left(\theta_{0}, \theta_{1}\right)\right| \\
& \leq \sum_{i=n}^{\infty} 2^{t}\left|\wp_{c b}\left(\theta_{0}, \theta_{1}\right)\right| \\
& =\frac{2^{n}}{1-\imath}\left|\wp_{c b}\left(\theta_{0}, \theta_{1}\right)\right| .
\end{aligned}
$$

Hence, we have

$$
\left|\wp_{c b}\left(\theta_{n}, \theta_{m}\right)\right| \leq \frac{2^{n}}{1-2}\left|\wp_{c b}\left(\theta_{0}, \theta_{1}\right)\right| \rightarrow 0 \quad \text { as } \quad n \rightarrow \infty .
$$

Hence, $\left\{\theta_{n}\right\}$ is a Cauchy sequence in $G$. In all cases above discussed, we get the sequence $\left\{\theta_{n}\right\}_{n \in \mathbb{N}}$, which is a Cauchy sequence. Since $G$ is complete, there exists $\theta^{*} \in G$ such that $\theta_{n} \rightarrow \theta^{*}$ as $n \rightarrow \infty$ and

$$
\wp_{c b}\left(\theta^{*}, \theta^{*}\right)=\lim _{n \rightarrow \infty} \wp_{c b}\left(\theta^{*}, \theta_{n}\right)=\lim _{n \rightarrow \infty} \wp_{c b}\left(\theta_{n}, \theta_{n}\right)=0
$$

By the continuity of $\Pi$, it follows that $\theta_{2 n+1}=\Pi \theta_{2 n} \rightarrow \Pi \theta^{*}$ as $n \rightarrow \infty$.

$$
\text { i.e., } \wp_{c b}\left(\Pi \theta^{*}, \Pi \theta^{*}\right)=\lim _{n \rightarrow \infty} \wp_{c b}\left(\Pi \theta^{*}, \Pi \theta_{2 n}\right)=\lim _{n \rightarrow \infty} \wp_{c b}\left(\Pi \theta_{2 n}, \Pi \theta_{2 n}\right) \text {. }
$$

However,

$$
\wp_{c b}\left(\Pi \theta^{*}, \Pi \theta^{*}\right)=\lim _{n \rightarrow \infty} \wp_{c b}\left(\Pi \theta_{2 n}, \Pi \theta_{2 n}\right)=\lim _{n \rightarrow \infty} \wp_{c b}\left(\theta_{2 n+1}, \theta_{2 n+1}\right)=0 .
$$

Next, we have to prove that $\theta^{*}$ is a fixed point of $\Pi$.

$$
\wp_{c b}\left(\Pi \theta^{*}, \theta^{*}\right) \preceq \wp_{c b}\left(\Pi \theta^{*}, \Pi \theta_{2 n}\right)+\wp_{c b}\left(\Pi \theta_{2 n}, \theta^{*}\right)-\wp_{c b}\left(\Pi \theta_{2 n}, \Pi \theta_{2 n}\right) .
$$


As $n \rightarrow \infty$, we obtain $\left|\wp_{c b}\left(\Pi \theta^{*}, \theta^{*}\right)\right| \leq 0$. Thus, $\wp_{c p}\left(\Pi \theta^{*}, \theta^{*}\right)=0$. Hence $\wp_{c b}\left(\theta^{*}, \theta^{*}\right)=$ $\wp_{c b}\left(\theta^{*}, \Pi \theta^{*}\right)=\wp_{c b}\left(\Pi \theta^{*}, \Pi \theta^{*}\right)=0$ and $\Pi \theta^{*}=\theta^{*}$. In the same way, we have $\theta^{*} \in G$ such that $\theta_{n} \rightarrow \theta^{*}$ as $n \rightarrow \infty$ and

$$
\wp_{c b}\left(\theta^{*}, \theta^{*}\right)=\lim _{n \rightarrow \infty} \wp_{c b}\left(\theta^{*}, \theta_{n}\right)=\lim _{n \rightarrow \infty} \wp_{c b}\left(\theta_{n}, \theta_{n}\right)=0
$$

By the continuity of $\Pi$, it follows $\theta_{2 n+2}=\Psi \theta_{2 n+1} \rightarrow \Psi \theta^{*}$ as $n \rightarrow \infty$.

$$
\text { i.e., } \wp_{c b}\left(\Psi \theta^{*}, \Psi \theta^{*}\right)=\lim _{n \rightarrow \infty} \wp_{c b}\left(\Psi \theta^{*}, \Psi \theta_{2 n+1}\right)=\lim _{n \rightarrow \infty} \wp_{c b}\left(\Psi \theta_{2 n+1}, \Psi \theta_{2 n+1}\right) .
$$

However,

$$
\wp_{c b}\left(\Psi \theta^{*}, \Psi \theta^{*}\right)=\lim _{n \rightarrow \infty} \wp_{c b}\left(\Psi \theta_{2 n+1}, \Psi \theta_{2 n+1}\right)=\lim _{n \rightarrow \infty} \wp_{c b}\left(\theta_{2 n+2}, \theta_{2 n+2}\right)=0 .
$$

Next we have to prove that $\theta^{*}$ is a fixed point of $\Psi$.

$$
\wp_{c b}\left(\Psi \theta^{*}, \theta^{*}\right) \preceq \wp_{c b}\left(\Psi \theta^{*}, \Psi \theta_{2 n+1}\right)+\wp_{c b}\left(\Psi \theta_{2 n+1}, \theta^{*}\right)-\wp_{c b}\left(\Psi \theta_{2 n+1}, \Pi \theta_{2 n+1}\right) .
$$

As $n \rightarrow \infty$, we obtain $\left|\wp_{c b}\left(\Psi \theta^{*}, \theta^{*}\right)\right| \leq 0$. Thus, $\wp_{c p}\left(\Psi \theta^{*}, \theta^{*}\right)=0$. Hence, $\wp_{c b}\left(\theta^{*}, \theta^{*}\right)=$ $\wp_{c b}\left(\theta^{*}, \Psi \theta^{*}\right)=\wp_{c b}\left(\Psi \theta^{*}, \Psi \theta^{*}\right)=0$ and $\Psi \theta^{*}=\theta^{*}$. Therefore, $\theta^{*}$ is a common fixed point of the pair $(\Pi, \Psi)$.

To prove uniqueness, let us consider $\omega^{*} \in G$ is another common fixed point for the pair $(\Pi, \Psi)$. Then

$$
\begin{aligned}
\wp_{c b}\left(\theta^{*}, \omega^{*}\right)= & \wp_{c b}\left(\Pi \theta^{*}, \Psi \omega^{*}\right) \\
\preceq & \curlywedge \max \left\{\wp_{c b}\left(\theta^{*}, \omega^{*}\right), \wp_{c b}\left(\theta^{*}, \Pi \theta^{*}\right), \wp_{c b}\left(\omega^{*}, \Psi \omega^{*}\right),\right. \\
& \left.\frac{1}{2}\left(\wp_{c b}\left(\theta^{*}, \Psi \omega^{*}\right)+\wp_{c b}\left(\omega^{*}, \Pi \theta^{*}\right)\right)\right\} \\
\preceq & \curlywedge \max \left\{\wp_{c b}\left(\theta^{*}, \omega^{*}\right), \wp_{c b}\left(\theta^{*}, \theta^{*}\right), \wp_{c b}\left(\omega^{*}, \omega^{*}\right),\right. \\
& \left.\frac{1}{2}\left(\wp_{c b}\left(\theta^{*}, \omega^{*}\right)+\wp_{c b}\left(\omega^{*}, \theta^{*}\right)\right)\right\} \\
\preceq & \curlywedge \wp_{c b}\left(\theta^{*}, \omega^{*}\right) .
\end{aligned}
$$

This implies that $\theta^{*}=\omega^{*}$.

In the absence of the continuity condition for the mappings $\Pi$ and $\Psi$, we get the the following theorem.

Theorem 3. Let $\left(G, \wp_{c b}\right)$ be a complete CPMS and $\Pi, \Psi: G \rightarrow G$ be two mappings such that

$$
\begin{aligned}
& \wp_{c b}(\Pi \theta, \Psi \omega) \preceq \curlywedge \max \left\{\wp_{c b}(\theta, \omega), \wp_{c b}(\theta, \Pi \theta), \wp_{c b}(\omega, \Psi \omega),\right. \\
&\left.\frac{1}{2}\left(\wp_{c b}(\theta, \Psi \omega)+\wp_{c b}(\omega, \Pi \theta)\right)\right\},
\end{aligned}
$$

for all $\theta, \omega \in G$, where $0 \leq \curlywedge<1$ and $\wp_{c b}(\Pi \theta, \Psi \omega) \neq 0$. Then, the pair $(\Pi, \Psi)$ has a unique common fixed point and $\wp_{c b}\left(\theta^{*}, \theta^{*}\right)=0$.

Proof. Following from Theorem 2, we get that the sequence $\left\{\theta_{n}\right\}$ is a Cauchy sequence. Since $G$ is complete, there exists $\theta^{*} \in G$ such that $\theta_{n} \rightarrow \theta^{*}$ as $n \rightarrow \infty$.

Since $\Pi$ and $\Psi$ are not continuous, we have $\wp_{c b}\left(\theta^{*}, \Pi \theta^{*}\right)=\vartheta>0$. 
Then, we estimate

$$
\begin{aligned}
\vartheta= & \wp_{c b}\left(\theta^{*}, \Pi \theta^{*}\right) \\
\preceq & \wp_{c b}\left(\theta^{*}, \theta_{2 i+2}\right)+\wp_{c b}\left(\theta_{2 i+2}, \Pi \theta^{*}\right)-\wp_{c b}\left(\theta_{2 i+2}, \theta_{2 i+2}\right) \\
\preceq & \wp_{c b}\left(\theta^{*}, \theta_{2 i+2}\right)+\wp_{c b}\left(\theta_{2 i+2}, \Pi \theta^{*}\right) \\
\preceq & \wp_{c b}\left(\theta^{*}, \theta_{2 i+2}\right)+\wp_{c b}\left(\Psi \theta_{2 i+1}, \Pi \theta^{*}\right) \\
\preceq & \wp_{c b}\left(\theta^{*}, \theta_{2 i+2}\right)+\curlywedge \max \left\{\wp_{c b}\left(\theta_{2 i+1}, \theta^{*}\right), \wp_{c b}\left(\theta_{2 i+1}, \Psi \theta_{2 i+1}\right), \wp_{c b}\left(\theta^{*}, \Pi \theta^{*}\right),\right. \\
& \left.\frac{1}{2}\left(\wp_{c b}\left(\theta_{2 i+1}, \Pi \theta^{*}\right)+\wp_{c b}\left(\theta^{*}, \Psi \theta_{2 i+1}\right)\right)\right\} \\
\preceq & \wp_{c b}\left(\theta^{*}, \theta_{2 i+2}\right)+\curlywedge \max \left\{\wp_{c b}\left(\theta_{2 i+1}, \theta^{*}\right), \wp_{c b}\left(\theta_{2 i+1}, \theta_{2 i+2}\right), \wp_{c b}\left(\theta^{*}, \Pi \theta^{*}\right),\right. \\
& \left.\frac{1}{2}\left(\wp_{c b}\left(\theta_{2 i+1}, \Pi \theta^{*}\right)+\wp_{c b}\left(\theta^{*}, \theta_{2 i+2}\right)\right)\right\} \\
\preceq & \wp_{c b}\left(\theta^{*}, \theta_{2 i+2}\right)+\curlywedge \wp_{c b}\left(\theta^{*}, \Pi \theta^{*}\right) \\
\preceq & s \wp_{c b}\left(\theta^{*}, \theta_{2 i+2}\right)+\curlywedge \vartheta .
\end{aligned}
$$

This yields

$$
|\vartheta| \leq\left|\wp_{c b}\left(\theta^{*}, \theta_{2 i+2}\right)\right|+\curlywedge|\vartheta|
$$

By definition,

$$
\lim _{i} \wp_{c b}\left(\theta^{*}, \theta_{2 i+2}\right)=\wp_{c b}\left(\theta^{*}, \theta^{*}\right)
$$

From the Cauchy property of $\left(\theta_{n}\right)$, the above limit is zero, and then,

$$
|\vartheta| \leq \curlywedge|\vartheta|
$$

Hence, $\curlywedge \geq 1$, which is a contradiction. Then $\theta^{*}=\Pi \theta^{*}$. In the same way, we obtain $\theta^{*}=\Psi \theta^{*}$. Hence $\theta^{*}$ is a common fixed point for the pair $(\Pi, \Psi)$ and $\wp_{c b}\left(\theta^{*}, \theta^{*}\right)=$ $\wp_{c b}\left(\theta^{*}, \Psi \theta^{*}\right)=\wp_{c b}\left(\Psi \theta^{*}, \Psi \theta^{*}\right)=0$. Uniqueness of the common fixed point $\theta^{*}$ follows from Theorem 2.

For $\Pi=\Psi$, we get the following fixed points results on CPMS.

Theorem 4. Let $\left(G, \wp_{c b}\right)$ be a complete CPMS and $\Pi: G \rightarrow G$ be a continuous mapping such that

$$
\begin{aligned}
& \wp_{c b}(\Pi \theta, \Pi \omega) \preceq \curlywedge \max \left\{\wp_{c b}(\theta, \omega), \wp_{c b}(\theta, \Pi \theta), \wp_{c b}(\omega, \Pi \omega),\right. \\
&\left.\frac{1}{2}\left(\wp_{c b}(\theta, \Pi \omega)+\wp_{c b}(\omega, \Pi \theta)\right)\right\},
\end{aligned}
$$

for all $\theta, \omega \in G$, where $0 \leq \curlywedge<1$ and $\wp_{c b}(\Pi \theta, \Pi \omega) \neq 0$. Then the pair $\Pi$ has a unique fixed point and $\wp_{c b}\left(\theta^{*}, \theta^{*}\right)=0$.

Remark 1. Similarly, we get a fixed point result in the absence of continuity condition for the mapping $\Pi$.

Corollary 1. Let $\left(G, \wp_{c b}\right)$ be a complete CPMS and $\Psi: G \rightarrow G$ be a continuous mapping such that

$$
\begin{aligned}
& \wp_{c b}\left(\Psi^{n} \theta, \Psi^{n} \omega\right) \preceq \curlywedge \max \left\{\wp_{c b}(\theta, \omega), \wp_{c b}\left(\theta, \Psi^{n} \theta\right), \wp_{c b}\left(\omega, \Psi^{n} \omega\right),\right. \\
&\left.\frac{1}{2}\left(\wp_{c b}\left(\theta, \Psi^{n} \omega\right)+\wp_{c b}\left(\omega, \Psi^{n} \theta\right)\right)\right\},
\end{aligned}
$$

for all $\theta, \omega \in G$, where $0 \leq \curlywedge<1, \wp_{c b}\left(\Psi^{n} \theta, \Psi^{n} \omega\right) \neq 0$ and $n \in \mathbb{N}$. Then, $\Psi$ has a unique fixed point and $\wp_{c b}\left(\theta^{*}, \theta^{*}\right)=0$. 
Proof. By Theorem 2, we get $\theta^{*} \in G$ such that $\Psi^{n} \theta^{*}=\theta^{*}$ and $\wp_{c b}\left(\theta^{*}, \theta^{*}\right)=0$. Then, we get

$$
\begin{aligned}
\wp_{c b}\left(\Psi \theta^{*}, \theta^{*}\right)= & \wp_{c b}\left(\Psi \Psi^{n} \theta^{*}, \Psi^{n} \theta^{*}\right)=\wp_{c b}\left(\Psi^{n} \Psi \theta^{*}, \Psi^{n} \theta^{*}\right) \\
\preceq & \curlywedge \max \left\{\wp_{c b}\left(\Psi \theta^{*}, \theta^{*}\right), \wp_{c b}\left(\Psi \theta^{*}, \Psi^{n} \Psi \theta^{*}\right), \wp_{c b}\left(\theta^{*}, \Psi^{n} \theta^{*}\right),\right. \\
& \left.\frac{1}{2}\left(\wp_{c b}\left(\Psi \theta^{*}, \Psi^{n} \theta^{*}\right)+\wp_{c b}\left(\theta^{*}, \Psi^{n} \Psi \theta^{*}\right)\right)\right\} \\
\preceq & \curlywedge \max \left\{\wp_{c b}\left(\Psi \theta^{*}, \theta^{*}\right), \wp_{c b}\left(\Psi \theta^{*}, \Psi \theta^{*}\right), \wp_{c b}\left(\theta^{*}, \theta^{*}\right),\right. \\
& \left.\frac{1}{2}\left(\wp_{c b}\left(\Psi \theta^{*}, \theta^{*}\right)+\wp_{c b}\left(\theta^{*}, \Psi \theta^{*}\right)\right)\right\} \\
= & \curlywedge \wp_{c b}\left(\Psi \theta^{*}, \theta^{*}\right) .
\end{aligned}
$$

Hence $\Psi^{n} \theta^{*}=\Psi \theta^{*}=\theta^{*}$. Then $\Psi$ has a unique fixed point.

Remark 2. From the above Corollary 1, similarly, we get a fixed-point result in the absence of continuity condition for the mapping $\Psi$.

Next, we present a new generalization of a common fixed point theorem on CPMS.

Theorem 5. Let $\left(G, \wp_{c b}\right)$ be a complete CPMS and $\Pi, \Psi: G \rightarrow G$ be two continuous mappings such that

$$
\wp_{c b}(\Pi \theta, \Psi \omega) \preceq \curlywedge \max \left\{\wp_{c b}(\theta, \omega), \frac{\wp_{c b}(\theta, \Pi \theta) \wp_{c b}(\omega, \Psi \omega)}{1+\wp_{c b}(\theta, \omega)}, \frac{\wp_{c b}(\theta, \Pi \theta) \wp_{c b}(\Pi \theta, \Psi \omega)}{1+\wp_{c b}(\theta, \omega)}\right\},
$$

for all $\theta, \omega \in G$, where $0 \leq \curlywedge<1$ and $\wp_{c b}(\Pi \theta, \Psi \omega) \neq 0$. Then, the pair $(\Pi, \Psi)$ has a unique common fixed point and $\wp_{c b}\left(\theta^{*}, \theta^{*}\right)=0$.

Proof. Let $\theta_{0}$ be arbitrary point in $G$ and define a sequence $\left\{\theta_{n}\right\}$ as follows:

$$
\theta_{2 n+1}=\Pi \theta_{2 n} \text { and } \theta_{2 n+2}=\Psi \theta_{2 n+1}, n=0,1,2, \ldots
$$

Then, by (12) and (13), we obtain

$$
\begin{aligned}
& \wp_{c b}\left(\theta_{2 n+1}, \theta_{2 n+2}\right)= \wp_{c b}\left(\Pi \theta_{2 n}, \Psi \theta_{2 n+1}\right) \\
& \preceq \curlywedge \max \left\{\wp_{c b}\left(\theta_{2 n}, \theta_{2 n+1}\right), \frac{\wp_{c b}\left(\theta_{2 n}, \theta_{2 n+1}\right) \wp_{c b}\left(\Psi \theta_{2 n+1}, \Pi \theta_{2 n}\right)}{1+\wp_{c b}\left(\theta_{2 n}, \theta_{2 n+1}\right)},\right. \\
&\left.\frac{\wp_{c b}\left(\theta_{2 n}, \Pi \theta_{2 n}\right) \wp_{c b}\left(\Pi \theta_{2 n}, \Psi \theta_{2 n+1}\right)}{1+\wp_{c b}\left(\theta_{2 n}, \theta_{2 n+1}\right)}\right\} \\
& \preceq \curlywedge \max \left\{\wp_{c b}\left(\theta_{2 n}, \theta_{2 n+1}\right), \frac{\wp_{c b}\left(\theta_{2 n}, \theta_{2 n+1}\right) \wp_{c b}\left(\theta_{2 n+1}, \theta_{2 n+2}\right)}{1+\wp_{c b}\left(\theta_{2 n}, \theta_{2 n+1}\right)},\right. \\
&\left.\frac{\wp_{c b}\left(\theta_{2 n}, \theta_{2 n+1}\right) \wp_{c b}\left(\theta_{2 n+1}, \theta_{2 n+2}\right)}{1+\wp_{c b}\left(\theta_{2 n}, \theta_{2 n+1}\right)}\right\} \\
& \preceq \curlywedge \max \left\{\wp_{c b}\left(\theta_{2 n}, \theta_{2 n+1}\right), \wp_{c b}\left(\theta_{2 n+1}, \theta_{2 n+2}\right)\right\} . \\
& \text { If } \max \left\{\wp_{c b}\left(\theta_{2 n}, \theta_{2 n+1}\right), \wp_{c b}\left(\theta_{2 n+1}, \theta_{2 n+2}\right)\right\}=\wp_{c b}\left(\theta_{2 n+1}, \theta_{2 n+2}\right), \text { then } \\
& \wp_{c b}\left(\theta_{2 n+1}, \theta_{2 n+2}\right) \preceq \curlywedge \wp_{c b}\left(\theta_{2 n+1}, \theta_{2 n+2}\right) .
\end{aligned}
$$

This shows that $\curlywedge \geq 1$, which is a contradiction. Therefore

$$
\wp_{c b}\left(\theta_{2 n+1}, \theta_{2 n+2}\right) \preceq \curlywedge \wp_{c b}\left(\theta_{2 n}, \theta_{2 n+1}\right) .
$$

Similarly, we obtain

$$
\wp_{c b}\left(\theta_{2 n+2}, \theta_{2 n+3}\right) \preceq \curlywedge \wp_{c b}\left(\theta_{2 n+1}, \theta_{2 n+2}\right) .
$$


From (14) and (15), $\forall n=0,1,2, \ldots$, we get

$$
\wp_{c b}\left(\theta_{n+1}, \theta_{n+2}\right) \preceq \curlywedge \wp_{c b}\left(\theta_{n}, \theta_{n+1}\right) \preceq \ldots \preceq \curlywedge^{n+1} \wp_{c b}\left(\theta_{0}, \theta_{1}\right) .
$$

For $m, n \in \mathbb{N}$, with $m>n$, we have

$$
\begin{aligned}
& \wp_{c b}\left(\theta_{n}, \theta_{m}\right) \preceq \wp_{c b}\left(\theta_{n}, \theta_{n+1}\right)+\wp_{c b}\left(\theta_{n+1}, \theta_{m}\right)-\wp_{c b}\left(\theta_{n+1}, \theta_{n+1}\right) \\
& \preceq \wp_{c b}\left(\theta_{n}, \theta_{n+1}\right)+\wp_{c b}\left(\theta_{n+1}, \theta_{m}\right) \\
& \preceq \wp_{c b}\left(\theta_{n}, \theta_{n+1}\right)+\wp_{c b}\left(\theta_{n+1}, \theta_{n+2}\right)+\wp_{c b}\left(\theta_{n+2}, \theta_{m}\right) \\
& -\wp_{c b}\left(\theta_{n+2}, \theta_{n+2}\right) \\
& \preceq \wp_{c b}\left(\theta_{n}, \theta_{n+1}\right)+\wp_{c b}\left(\theta_{n+1}, \theta_{n+2}\right)+\wp_{c b}\left(\theta_{n+2}, \theta_{m}\right) \\
& \preceq \wp_{c b}\left(\theta_{n}, \theta_{n+1}\right)+\wp_{c b}\left(\theta_{n+1}, \theta_{n+2}\right)+\wp_{c b}\left(\theta_{n+2}, \theta_{n+3}\right) \\
& +\ldots+\wp_{c b}\left(\theta_{m-2}, \theta_{m-1}\right)+s^{m-n} \wp_{c b}\left(\theta_{m-1}, \theta_{m}\right) \text {. }
\end{aligned}
$$

By using (16), we get

$$
\begin{aligned}
\wp_{c b}\left(\theta_{n}, \theta_{m}\right) \preceq & \curlywedge^{n} \wp_{c b}\left(\theta_{0}, \theta_{1}\right)+\curlywedge^{n+1} \wp_{c b}\left(\theta_{0}, \theta_{1}\right)+\curlywedge^{n+2} \wp_{c b}\left(\theta_{0}, \theta_{1}\right) \\
& +\ldots+\curlywedge^{m-2} \wp_{c b}\left(\theta_{0}, \theta_{1}\right)+\curlywedge^{m-1} \wp_{c b}\left(\theta_{0}, \theta_{1}\right) \\
= & \sum_{i=1}^{m-n} \curlywedge^{i+n-1} \wp_{c b}\left(\theta_{0}, \theta_{1}\right) .
\end{aligned}
$$

Therefore,

$$
\begin{aligned}
\left|\wp_{c b}\left(\theta_{n}, \theta_{m}\right)\right| & \leq \sum_{i=1}^{m-n} \curlywedge^{i+n-1}\left|\wp_{c b}\left(\theta_{0}, \theta_{1}\right)\right|=\sum_{i=1}^{m-n} \curlywedge^{t}\left|\wp_{c b}\left(\theta_{0}, \theta_{1}\right)\right| \\
& \leq \sum_{i=n}^{\infty} \curlywedge^{t}\left|\wp_{c b}\left(\theta_{0}, \theta_{1}\right)\right| \\
& =\frac{\curlywedge^{n}}{1-\curlywedge^{n}}\left|\wp_{c b}\left(\theta_{0}, \theta_{1}\right)\right| .
\end{aligned}
$$

Hence, we have

$$
\left|\wp_{c b}\left(\theta_{n}, \theta_{m}\right)\right| \leq \frac{\curlywedge^{n}}{1-\curlywedge}\left|\wp_{c b}\left(\theta_{0}, \theta_{1}\right)\right| \rightarrow 0 \quad \text { as } \quad n \rightarrow \infty .
$$

Hence, $\left\{\theta_{n}\right\}$ is a Cauchy sequence in $G$. Since $G$ is complete, there exists $\theta^{*} \in G$ such that $\theta_{n} \rightarrow \theta^{*}$ as $n \rightarrow \infty$ and

$$
\wp_{c b}\left(\theta^{*}, \theta^{*}\right)=\lim _{n \rightarrow \infty} \wp_{c b}\left(\theta^{*}, \theta_{n}\right)=\lim _{n \rightarrow \infty} \wp_{c b}\left(\theta_{n}, \theta_{n}\right)=0
$$

Since $\Psi$ is continuous, it yields

$$
\theta^{*}=\lim _{n \rightarrow \infty} \theta_{2 n+2}=\lim _{n \rightarrow \infty} \Psi \theta_{2 n+1}=\Psi \lim _{n \rightarrow \infty} \theta_{2 n+1}=\Psi \theta^{*} .
$$

Similarly, by the continuity of $\Pi$, we get $\theta^{*}=\Pi \theta^{*}$. Then the pair $(\Pi, \Psi)$ has a common fixed point. To prove uniqueness, let us consider that $\omega^{*} \in G$ is another common fixed point for the pair $(\Pi, \Psi)$. Then 


$$
\begin{aligned}
\wp_{c b}\left(\theta^{*}, \omega^{*}\right)= & \wp_{c b}\left(\Pi \theta^{*}, \Psi \omega^{*}\right) \\
\preceq & \curlywedge \max \left\{\wp_{c b}\left(\theta^{*}, \omega^{*}\right), \frac{\wp_{c b}\left(\theta^{*}, \Pi \theta^{*}\right) \wp_{c b}\left(\omega^{*}, \Psi \omega^{*}\right)}{1+\wp_{c b}\left(\theta^{*}, \omega^{*}\right)},\right. \\
& \left.\frac{\wp_{c b}\left(\theta^{*}, \Pi \theta^{*}\right) \wp_{c b}\left(\Psi \omega^{*}, \Pi \theta^{*}\right)}{1+\wp_{c b}\left(\theta^{*}, \omega^{*}\right)}\right\} \\
\preceq & \wp_{c b}\left(\theta^{*}, \omega^{*}\right)
\end{aligned}
$$

This implies that $\theta^{*}=\omega^{*}$.

In the absence of the continuity condition for the mapping $\Pi$ and $\Psi$ in the Theorem 5, we obtain the following result.

Theorem 6. Let $\left(G, \wp_{c b}\right)$ be a complete $C P M S$ and $\Pi, \Psi: G \rightarrow G$ be two mappings such that

$$
\wp_{c b}(\Pi \theta, \Psi \omega) \preceq \curlywedge \max \left\{\wp_{c b}(\theta, \omega), \frac{\wp_{c b}(\theta, \Pi \theta) \wp_{c b}(\omega, \Psi \omega)}{1+\wp_{c b}(\theta, \omega)}, \frac{\wp_{c b}(\theta, \Pi \theta) \wp_{c b}(\Pi \theta, \Psi \omega)}{1+\wp_{c b}(\theta, \omega)}\right\},
$$

for all $\theta, \omega \in G$, where $0 \leq \curlywedge<1$ and $\wp_{c b}(\Pi \theta, \Psi \omega) \neq 0$. Then the pair $(\Pi, \Psi)$ has a unique common fixed point and $\wp_{c b}\left(\theta^{*}, \theta^{*}\right)=0$.

Proof. Following from Theorem 5 , we get that the sequence $\left\{\theta_{n}\right\}$ is a Cauchy sequence. Since $G$ is complete, then there exists $\theta^{*} \in G$ such that $\theta_{n} \rightarrow \theta^{*}$ as $n \rightarrow \infty$ and

$$
\wp_{c b}\left(\theta^{*}, \theta^{*}\right)=\lim _{n \rightarrow \infty} \wp_{c b}\left(\theta^{*}, \theta_{n}\right)=\lim _{n \rightarrow \infty} \wp_{c b}\left(\theta_{n}, \theta_{n}\right)=0 .
$$

Since $\Pi$ and $\Psi$ are not continuous, we have $\wp_{c b}\left(\theta^{*}, \Pi \theta^{*}\right)=\vartheta>0$.

Then, we estimate

$$
\begin{aligned}
& \vartheta=\wp_{c b}\left(\theta^{*}, \Pi \theta^{*}\right) \\
& \preceq \wp_{c b}\left(\theta^{*}, \theta_{2 i+2}\right)+\wp_{c b}\left(\theta_{2 i+2}, \Pi \theta^{*}\right)-\wp_{c b}\left(\theta_{2 i+2}, \theta_{2 i+2}\right) \\
& \preceq \wp_{c b}\left(\theta^{*}, \theta_{2 i+2}\right)+\wp_{c b}\left(\Pi \theta^{*}, \theta_{2 i+2}\right) \\
& \preceq \wp_{c b}\left(\theta^{*}, \theta_{2 i+2}\right)+\wp_{c b}\left(\Pi \theta^{*}, \Psi \theta_{2 i+1}\right) \\
& \preceq \wp_{c b}\left(\theta^{*}, \theta_{2 i+2}\right)+\curlywedge \max \left\{\wp_{c b}\left(\theta^{*}, \theta_{2 i+1}\right), \frac{\wp_{c b}\left(\theta^{*}, \Pi \theta^{*}\right) \wp_{c b}\left(\theta_{2 i+1}, \Psi \theta_{2 i+1}\right)}{1+\wp_{c b}\left(\theta^{*}, \theta_{2 i+1}\right)},\right. \\
& \left.\frac{\wp_{c b}\left(\theta^{*}, \Pi \theta_{*}\right) \wp_{c b}\left(\Pi \theta^{*}, \Psi \theta_{2 i+1}\right)}{1+\wp_{c b}\left(\theta^{*}, \theta_{2 i+1}\right)}\right\} \\
& \preceq \wp_{c b}\left(\theta^{*}, \theta_{2 i+2}\right)+\curlywedge \max \left\{\wp_{c b}\left(\theta^{*}, \theta_{2 i+1}\right), \frac{\wp_{c b}\left(\theta^{*}, \Pi \theta^{*}\right) \wp_{c b}\left(\theta_{2 i+1}, \theta_{2 i+2}\right)}{1+\wp_{c b}\left(\theta^{*}, \theta_{2 i+1}\right)}\right\}, \\
& \left.\frac{\wp_{c b}\left(\theta^{*}, \Pi \theta^{*}\right) \wp_{c b}\left(\Pi \theta^{*}, \theta_{2 i+2}\right)}{1+\wp_{c b}\left(\theta^{*}, \theta_{2 i+1}\right)}\right\} \\
& \preceq \wp_{c b}\left(\theta^{*}, \theta_{2 i+2}\right)+\curlywedge \wp_{c b}\left(\theta^{*}, \Pi \theta^{*}\right)^{2} \\
& \preceq \wp_{c b}\left(\theta^{*}, \theta_{2 i+2}\right)+\curlywedge \vartheta^{2} \text {. }
\end{aligned}
$$

This yields

$$
|\vartheta| \leq\left|\wp_{c b}\left(\theta^{*}, \theta_{2 i+2}\right)\right|+\curlywedge|\vartheta|^{2} .
$$

Hence, $\curlywedge \geq 1$, which is a contradiction. Then $\theta^{*}=\Pi \theta^{*}$. In the same way, we obtain $\theta^{*}=\Psi \theta^{*}$. Hence, $\theta^{*}$ is a common fixed point for the pair $(\Pi, \Psi)$. For uniqueness of the common fixed point, $\theta^{*}$ follows from Theorem 5 .

For $\Pi=\Psi$, we get the following fixed-points results on CPMS. 
Theorem 7. Let $\left(G, \wp_{c b}\right)$ be a complete CPMS and $\Pi: G \rightarrow G$ be a continuous mapping such that

$$
\wp_{c b}(\Pi \theta, \Pi \omega) \preceq \curlywedge \max \left\{\wp_{c b}(\theta, \omega), \frac{\wp_{c b}(\theta, \Pi \theta) \wp_{c b}(\omega, \Pi \omega)}{1+\wp_{c b}(\theta, \omega)}, \frac{\wp_{c b}(\theta, \Pi \theta) \wp_{c b}(\Pi \theta, \Pi \omega)}{1+\wp_{c b}(\theta, \omega)}\right\},
$$

for all $\theta, \omega \in G$, where $0 \leq \curlywedge<1$ and $\wp_{c b}(\Pi \theta, \Pi \omega) \neq 0$. Then, $\Pi$ has a unique fixed point and $\wp_{c b}\left(\theta^{*}, \theta^{*}\right)=0$.

Remark 3. Similarly, in the absence of continuity condition, we can get a fixed point result on $\Pi$.

Corollary 2. Let $\left(G, \wp_{c b}\right)$ be a complete CPMS and $\Pi: G \rightarrow G$ be a continuous mapping such that

$$
\begin{aligned}
\wp_{c b}\left(\Pi^{n} \theta, \Pi^{n} \omega\right) \preceq & \curlywedge \max \left\{\wp_{c b}(\theta, \omega), \frac{\wp_{c b}\left(\theta, \Pi^{n} \theta\right) \wp_{c b}\left(\omega, \Pi^{n} \omega\right)}{1+\wp_{c b}(\theta, \omega)},\right. \\
& \left.\frac{\wp_{c b}\left(\theta, \Pi^{n} \theta\right) \wp_{c b}\left(\Pi^{n} \theta, \Pi \omega\right)}{1+\wp_{c b}(\theta, \omega)}\right\},
\end{aligned}
$$

for all $\theta, \omega \in G$, where $0 \leq \curlywedge<1$ and $\wp_{c b}\left(\Pi^{n} \theta, \Pi^{n} \omega\right) \neq 0$. Then $\Pi$ has a unique fixed point and $\wp_{c b}\left(\theta^{*}, \theta^{*}\right)=0$.

Proof. By Theorem 5 , we get $\theta^{*} \in G$ such that $\Pi^{n} \theta^{*}=\theta^{*}$ and $\wp_{c b}\left(\theta^{*}, \theta^{*}\right)=0$. Then we get

$$
\begin{aligned}
\wp_{c b}\left(\Pi \theta^{*}, \theta^{*}\right)= & \wp_{c b}\left(\Pi \Pi^{n} \theta^{*}, \Pi^{n} \theta^{*}\right)=\wp_{c b}\left(\Pi^{n} \Pi \theta^{*}, \Pi^{n} \theta^{*}\right) \\
\preceq & \curlywedge \max \left\{\wp_{c b}\left(\Pi \theta^{*}, \theta^{*}\right), \frac{\wp_{c b}\left(\Pi \theta^{*}, \Pi^{n} \Pi \theta^{*}\right) \wp_{c b}\left(\theta^{*}, \Pi^{n} \theta^{*}\right)}{1+\wp_{c b}\left(\Pi \theta^{*}, \theta^{*}\right)},\right. \\
& \left.\frac{\wp_{c b}\left(\Pi \theta^{*}, \Pi^{n} \Pi \theta^{*}\right) \wp_{c b}\left(\Pi^{n} \Pi \theta^{*}, \Pi^{n} \theta^{*}\right)}{1+\wp_{c b}\left(\Pi \theta^{*}, \theta^{*}\right)}\right\} \\
\preceq & \curlywedge \max \left\{\wp_{c b}\left(\Pi \theta^{*}, \theta^{*}\right), \frac{\wp_{c b}\left(\Pi \theta^{*}, \Pi \Pi^{n} \theta^{*}\right) \wp_{c b}\left(\theta^{*}, \Pi^{n} \theta^{*}\right)}{1+\wp_{c b}\left(\Pi \theta^{*}, \theta^{*}\right)},\right. \\
& \left.\frac{\wp_{c b}\left(\Pi \theta^{*}, \Pi \Pi^{n} \theta^{*}\right) \wp_{c b}\left(\Pi \Pi^{n} \theta^{*}, \Pi^{n} \theta^{*}\right)}{1+\wp_{c b}\left(\Pi \theta^{*}, \theta^{*}\right)}\right\} \\
= & \curlywedge \wp_{c b}\left(\Pi \theta^{*}, \theta^{*}\right) .
\end{aligned}
$$

Hence $\Pi^{n} \theta^{*}=\Pi \theta^{*}=\theta^{*}$. Then, $\Pi$ has a unique fixed point.

Remark 4. From the above corollary 2, similarly, we get a fixed point result in the absence of continuity condition for the mapping $\Pi$.

Example 1. Let $G=\{1,2,3,4\}$ be endowed with the order $\theta \preceq \omega$ if and only if $\theta \leq \omega$. Then, $\preceq$ is a partial order in $G$. Define the complex partial metric space $\wp_{c b}: G \times G \rightarrow \mathbb{C}^{+}$as follows:

\begin{tabular}{|l|l|}
\hline$(\theta, \omega)$ & $\wp_{c b}(\theta, \omega)$ \\
\hline$(1,1),(2,2)$ & 0 \\
\hline$(1,2),(2,1),(1,3),(3,1),(2,3),(3,2),(3,3)$ & $e^{i x}$ \\
\hline$(1,4),(4,1),(2,4),(4,2),(3,4),(4,3),(4,4)$ & $3 e^{i x}$ \\
\hline
\end{tabular}

Obviously, $\left(G, \wp_{c b}\right)$ is a complete CPMS for $x \in\left[0, \frac{\pi}{2}\right]$. Define $\Pi, \Psi: G \rightarrow G$ by $\Pi \theta=1$,

$$
\Psi(\theta)= \begin{cases}1 & \text { if } \theta \in\{1,2,3\} \\ 2 & \text { if } \theta=4\end{cases}
$$

Clearly $\Pi$ and $\Psi$ are continuous functions. Now, for $\curlywedge=\frac{1}{3}$, we consider the following cases: (A) If $\theta=1$ and $\omega \in G-\{4\}$, then $\Pi(\theta)=\Psi(\omega)=1$ and the conditions of Theorem 2 are satisfied. 
(B) If $\theta=1, \omega=4$, then $\Pi \theta=1, \Psi \omega=2$,

$$
\begin{aligned}
\wp_{c b}(\Pi \theta, \Psi \omega)=e^{i x} \preceq & 3 \curlywedge e^{i x} \\
= & \curlywedge \max \left\{3 e^{i x}, 0,3 e^{i x}, \frac{1}{2}\left(e^{i x}+3 e^{i x}\right)\right\} \\
= & \curlywedge \max \left\{\wp_{c b}(\theta, \omega), \wp_{c b}(\theta, \Pi \theta), \wp_{c b}(\omega, \Psi \omega),\right. \\
& \left.\frac{1}{2}\left(\wp_{c b}(\theta, \Psi \omega)+\wp_{c b}(\omega, \Pi \theta)\right)\right\},
\end{aligned}
$$

(C) If $\theta=2, \omega=4$, then $\Pi \theta=1, \Psi \omega=2$,

$$
\begin{aligned}
\wp_{c b}(\Pi \theta, \Psi \omega)=e^{i x} \preceq & \preceq \curlywedge e^{i x} \\
= & \curlywedge \max \left\{3 e^{i x}, e^{i x}, 3 e^{i x}, \frac{1}{2}\left(0+3 e^{i x}\right)\right\} \\
= & \curlywedge \max \left\{\wp_{c b}(\theta, \omega), \wp_{c b}(\theta, \Pi \theta), \wp_{c b}(\omega, \Psi \omega),\right. \\
& \left.\frac{1}{2}\left(\wp_{c b}(\theta, \Psi \omega)+\wp_{c b}(\omega, \Pi \theta)\right)\right\},
\end{aligned}
$$

(D) If $\theta=3, \omega=4$, then $\Pi \theta=1, \Psi \omega=2$,

$$
\begin{aligned}
\wp_{c b}(\Pi \theta, \Psi \omega)=e^{i x} \preceq & 3 \curlywedge e^{i x} \\
= & \curlywedge \max \left\{3 e^{i x}, e^{i x}, 3 e^{i x}, \frac{1}{2}\left(e^{i x}+3 e^{i x}\right)\right\} \\
= & \curlywedge \max \left\{\wp_{c b}(\theta, \omega), \wp_{c b}(\theta, \Pi \theta), \wp_{c b}(\omega, \Psi \omega),\right. \\
& \left.\frac{1}{2}\left(\wp_{c b}(\theta, \Psi \omega)+\wp_{c b}(\omega, \Pi \theta)\right)\right\},
\end{aligned}
$$

(E) If $\theta=4, \omega=4$, then $\Pi \theta=2, \Psi \omega=2$,

$$
\begin{aligned}
\wp_{c b}(\Pi \theta, \Psi \omega)=e^{i x} \preceq & 3 \curlywedge e^{i x} \\
= & \curlywedge \max \left\{3 e^{i x}, 3 e^{i x}, 3 e^{i x}, \frac{1}{2}\left(3 e^{i x}+3 e^{i x}\right)\right\} \\
= & \curlywedge \max \left\{\wp_{c b}(\theta, \omega), \wp_{c b}(\theta, \Pi \theta), \wp_{c b}(\omega, \Psi \omega),\right. \\
& \left.\frac{1}{2}\left(\wp_{c b}(\theta, \Psi \omega)+\wp_{c b}(\omega, \Pi \theta)\right)\right\},
\end{aligned}
$$

Moreover, for $\curlywedge=\frac{1}{3}$, with $\curlywedge<1$, the conditions of Theorem 2 are satisfied. Therefore, 1 is the unique common fixed point of $\Pi$ and $\Psi$.

\section{Application}

Consider the following systems of nonlinear integral equations:

$$
w(s)=\mathfrak{F}(s)+\int_{a}^{b} T_{1}(s, p, w(p)) d p,
$$

and

$$
z(s)=\mathfrak{F}(s)+\int_{a}^{b} T_{2}(s, p, z(p)) d p,
$$

where

(i) $\quad \mathfrak{F}:[a, b] \rightarrow \mathbb{R}^{n}$ is a continuous mapping and $\mathfrak{F}(s)$ is a given function in $\left(C([a, b]), \mathbb{R}^{n}\right)$,

(ii) $\quad w(s)$ and $z(s)$ are unknown variables for each $s \in J=[a, b], b>a \geq 0$,

(iii) $\quad T_{1}(s, p)$ and $T_{2}(s, p)$ are deterministic kernels defined for $s, p \in J=[a, b]$. 
In this section, we present an existence theorem for a common solution to (18) and (19) that belongs to $G=\left(C(J), \mathbb{R}^{n}\right)$ (the set of continuous functions defined on $J$ ) by using the obtained result in Theorem 2. We consider the continuous mappings $\Pi, \Psi: G \rightarrow G$ given by

$$
\Pi w(s)=\mathfrak{F}(s)+\int_{a}^{b} T_{1}(s, p, w(p)) d p, w \in G, s \in J,
$$

and

$$
\Psi z(s)=\mathfrak{F}(s)+\int_{a}^{b} T_{2}(s, p, z(p)) d p, z \in G, s \in J,
$$

Then, the existence of a common solution to the nonlinear integral Equations (18) and (19) is equivalent to the existence of a common fixed point of $\Pi$ and $\Psi$. It is well known that $G$, endowed with the metric $\wp_{c b}$, defined by

$$
\wp_{c b}(w, z)=\sup _{s \in J}|w(s)-z(s)|+2,
$$

for all $w, z \in G$, is a complete CPMS. $G$ can also be equipped with the partial order $\preceq$ given by

$$
w, z \in G, w \preceq z \text { if and only } w(s) \geq z(s), \text { for all } s \in J .
$$

Further, let us consider a system of nonlinear integral equation as (18) and (19) under the following condition hold:

(A) $\quad T_{1}, T_{2}: J \times J \times \mathbb{R}^{n} \rightarrow \mathbb{R}^{n}$ are continuous functions satisfying

$$
\left|T_{1}(s, p, w(p))-T_{2}(s, p, z(p))\right| \preceq \frac{S(w, z)}{(b-a) e^{t}}-\frac{2}{b-a}, \forall t>0,
$$

where

$$
\begin{gathered}
S(w, z)=\max \left\{\wp_{c b}(w, z), \wp_{c b}(w, \Pi w), \wp_{c b}(z, \Psi z),\right. \\
\left.\frac{1}{2}\left(\wp_{c b}(w, \Psi z)+\wp_{c b}(z, \Pi w)\right)\right\} .
\end{gathered}
$$

Theorem 8. Let $\left(C(J), \mathbb{R}^{n}, \wp_{c b}\right)$ be a complete CPMS; then, the system (18) and (19) under condition $(A)$ have a unique common solution.

Proof. For $w, z \in\left(C(J), \mathbb{R}^{n}\right)$ and $s \in J$, we define the continuous mappings $\Pi, \Psi: G \rightarrow G$ by

$$
\Pi w(s)=\mathfrak{F}(s)+\int_{a}^{b} T_{1}(s, p, w(p)) d p,
$$

and

$$
\Psi z(s)=\mathfrak{F}(s)+\int_{a}^{b} T_{2}(s, p, z(p)) d p .
$$


Then, we have

$$
\begin{aligned}
\wp_{c b}(\Pi w(s), \Psi z(s)) & =\sup _{s \in J}|\Pi w(s)-\Psi z(s)|+2 \\
& \preceq \int_{a}^{b}\left|T_{1}(s, p, w(p))-T_{2}(s, p, z(p))\right| d p+2 \\
& \int_{a}^{b}\left(\frac{S(w, z)}{(b-a) e^{t}}-\frac{2}{b-a}\right) d p+2 \\
& =\frac{S(w, z)}{e^{t}} \\
& =\curlywedge S(w, z) \\
& =\curlywedge \max \left\{\wp_{c b}(w, z), \wp_{c b}(w, \Pi w), \wp_{c b}(z, \Psi z),\right. \\
& \left.\frac{1}{2}\left(\wp_{c b}(w, \Psi z)+\wp_{c b}(z, \Pi w)\right)\right\} .
\end{aligned}
$$

Hence, all the conditions of Theorem 2 are satisfied for $0<\curlywedge=\frac{1}{e^{t}}<1$ with $t>0$. Therefore the system of nonlinear integral Equations (18) and (19) have a unique common solution.

\section{Conclusions}

In this paper, we proved some common fixed-point theorems on complex partial metric space. An illustrative example and application on complex partial metric space is given.

Author Contributions: G.M., A.J.G., Y.L. and Z.G. contributed equally in writing this article. All authors read and approved the final manuscript.

Funding: This work was supported by the National Natural Science Foundation of P. R. China (Nos. 11971493 and 12071491).

Institutional Review Board Statement: Not applicable.

Informed Consent Statement: Not applicable.

Data Availability Statement: Not applicable.

Conflicts of Interest: The authors declare no conflict of interest.

\section{References}

1. Azam, A.; Fisher, B.; Khan, M. Common fixed point theorems in complex valued metric spaces. Numer. Funct. Anal. Optim. 2011, 32, 243-253. [CrossRef]

2. Rao, K.P.R.; Swamy, P.R.; Prasad, J.R. A common fixed point theorem in complex valued b-metric spaces. Bull. Math. Stat. Res. 2013, 1 .

3. Dhivya, P.; Marudai, M. Common fixed point theorems for mappings satisfying a contractive condition of rational expression on an ordered complex partial metric space. Cogent Math. 2017, 4, 1389622. [CrossRef]

4. Gunaseelan, M. Generalized fixed point theorems on complex partial b-metric space. Int. J. Res. Anal. Rev. 2019, 6.

5. Prakasam, L.M.; Gunaseelan, M. Common fixed point theorems using (CLR) and (EA) properties in complex partial b-metric space. Adv. Math. Sci. J. 2020, 1, 2773-2790. [CrossRef]

6. Gunaseelan, M.; Joseph, G.A.; Ramakrishnan, K.; Gaba, Y.U. Results on Complex Partial b-Metric Space with an Application. Math. Probl. Eng. 2021, 10, 2021. 